\title{
Prediction and Diversion Mechanisms for Crowd Management Based on Risk Rating
}

\author{
Meihua Zhang ${ }^{1,2}$, Yuan $\mathrm{Yao}^{3}$, Kefan Xie ${ }^{1}$ \\ ${ }^{1}$ School of Management, Wuhan University of Technology, Wuhan, China \\ ${ }^{2}$ School of Management, South-Central University for Nationalities, Wuhan, China \\ ${ }^{3}$ College of Physical Science and Technology, Central China Normal University, Wuhan, China \\ Email: meganzhang@mail.scuec.edu.cn
}

How to cite this paper: Zhang, M.H., Yao, Y. and Xie, K. (2017) Prediction and Diversion Mechanisms for Crowd Management Based on Risk Rating. Engineering, 9, 377387.

https://doi.org/10.4236/eng.2017.95021

Received: April 17, 2017

Accepted: May 24, 2017

Published: May 27, 2017

Copyright (c) 2017 by authors and Scientific Research Publishing Inc. This work is licensed under the Creative Commons Attribution International License (CC BY 4.0).

http://creativecommons.org/licenses/by/4.0/

\section{(c) (i) Open Access}

\begin{abstract}
Studies of past accidents have revealed that various elements such as failure to identify hazards, crowd behaviors out of controlling, deficiency of the egress signage system, inconsistency between process behavior and process plan, and environmental constraints, etc. affected crowd evacuation. Above all, the human factor is the key issue in safety and disaster management, although it is bound to other factors inextricably. This paper explores crowd behaviors that may influence an urgent situation, and discusses the technique applied to the crowd prediction. Based on risk rating relative to crowd density, risk plans for different levels are proposed to dispose the potential threats. Also practical crowd management measures at different risk levels are illustrated in a case of a metro station in China. Finally, the strategies for crowd security management are advised that all stakeholders are amenable to form risk consciousness and implement safety procedures consistent with risk plans professionally and scientifically.
\end{abstract}

\section{Keywords}

Crowd Security, Crowd Behaviors, Crowd Density, Risk Rating, Crowd

Diversion

\section{Introduction}

In recent years, disasters such as stampede accidents in public places have happened from time to time, which has resulted in a shock and taught us painful lessons. Numbers of participants aggregate on many occasions when various celebrations or sport events are held, or overcrowding occurs in public places from the subway stations to the city squares, which makes the security risk potentially accompanied. Practice has witnessed when the crowd density sharply 
increases to a certain degree, the overcrowding brings mutual influence and great pressure among people. In this case once someone falls, he may have no time to react and no place to stand upright. At that time people may be forced to step on others when trying to avoid the deadly "Domino Effect" that is falling down by the push from each other. The order of the crowds may break down when the number of participants reaches a certain point, which is not about the people's morality or quality, but is aroused by the normal interaction of people [1]. Or if the crowd flow converges at the bottleneck places, or reverse cluster and heterogeneous collection joined, stampede accidents are more likely to happen. It is difficult to clearly determine the responsibility that everyone has, and normal communication means cannot work [2]. Technique is rational while loss of emotional control in crowds is human nature. The "Civilized" people in the crowded situation are irritable, even offensive, and prone to misconduct, although other factors such as the defect of venue design and the fault decision of activity site management have been also proved to trigger the public security crisis [3]. Complex factors bring about the great difficulty of crowd security management in emergency events, moreover, the human factor, unsurprisingly, increases the event risk remarkably. Therefore, understanding human behavior in crowds, interpreting the context, predicting and deciding on the use of possible intervention mechanisms have become undisputedly indispensable for crowd security management.

Over the past decades, although crowd research and crowd management practice have developed and improved greatly to give support to crowd managers in preparation and during a crowd event, much more is needed [4]. For example, crowd behavior models grounded in science originate from different disciplines including psychology, sociology, theoretical physics, applied mathematics, artificial intelligence and computer science have been established to help stakeholders better understand crowd behavior, but only few have been actually used to assist in crowd management, maybe partly because crowd behaviors are of significant uncertainties while some models either coming in different forms or ranging from extremely formal or implicit knowledge are not ready for use [5]. Simultaneously, technological tools and proposals about crowd organization and venue design have been offered for particular aspects of crowd management, but most of them are not for usability in practice. A substantial gap between crowd research and crowd management practice is obvious, and more practical decision support approach is needed to provide interventions for crowd security management.

This paper attempts to explore how crowd management is practiced today by the application of a case study in a metro station in China. The structure is organized by first providing insights into the individuals' and the groups' behavior characteristics in Section 2. In Section 3, concentrating on crowd density, the relevant operation support such as its supervision, estimation and grade are discussed. Based on the analysis of crowd behaviors and the grade of crowd density, prediction and division strategies on crowd management are proposed in Sec- 
tion 4. Practical measures for crowd management at different risk levels are illustrated in a case of a metro station in China in Section 5. And then conclusions come in Section 6.

\section{Analysis of Crowd Behavior Characteristics}

Individuals' behaviors in the crowds have the characters of strong chance, randomness, and even loss of the volition to control themselves in the events. For example, normally people head smoothly in the beginning, and if a crowd makes individual movement slow and complex, they would be stop-and-go, and then chaos might occur as crowds became more dense [6]. Crowd behaviors have been represented in these three statements.

1) Crowd Panic and Herding Behaviors.

Unexpected incidents may sink crowds into the scare, or even lead to crowd panic which further results in emergency situation. At that time people's normal and conscious personality has been deeply influenced and often replaced by the unconscious and irrational behaviors unless there are certain strong social impacts occurring, such as leaders who can make decisions for the group, otherwise, individuals tend to give up their own opinions and follow others under the pressure in crowds [7]. In fact, it is so difficult for people to respond to all the information in the site to make correct judgments in the emergency that the conformity is easier to arise during the evacuation. Blind conformity may break the balance of the flow and cause crowd accidents and stampede accidents.

\section{2) Decision Theory and "Fast-Is-Slower" Effect.}

Decision theory assumes that persons in danger are able to make reasonable decisions (even if it is limited) and also attempt to get good results and goals in this case. However, the individual optimum behaviors always couldn't attain the best choice for the group, and even sometimes result in the worst. In a fire, for example, to cooperate with others and to queue up are most likely to benefit the entire group to escape, thus an individual chance to leave dangerous facilities increases. Unfortunately, if someone pushes, other individuals may feel that if they do not follow the push, their chance to escape will be threatened, so after consideration the individuals' action turns out to join the move competition, push others, and try to make every effort to get out of the dangerous area rapidly. If everyone moves fast, the effect of rubbing and squeezing will reduce their fleeing efficiency. The evacuation time shows a tendency of decrease in the beginning and then an increasing trend with the walkers' expecting speed increasing [8]. Consequently, the crowd should be suggested to calm down and make the escape orderly to eliminate the blind movement and avoid the Fast-Is-Slower Effect.

\section{3) Arching Phenomenon and "Domino Effect".}

The inlet, popular destinations and the outlet are indicated to be the bottleneck during people's movement. When a crowd gathers at these places, arching phenomenon may appear. For example, when in emergency a number of people try to escape simultaneously from the outlet, arching, clogging, and exit rate 
burst can be observed [9]. At this point the bottleneck places become so critical that once a tiny incident such as someone's falling, picking, or rumor occurs, the "Domino Effect" in congestion accompanies easily due to great interaction pressure transmitted among the congestion points. The width of bottleneck areas limits crowd to move quickly so that a jam arises. And the disaster may happen because of competitive behaviors. Three factors determine the congestion degree and the evacuation speed at the bottleneck: serious consequence resulted from failing to evacuate rapidly, the available evacuation time and the group size. Therefore, taking appropriate measures to reduce the number of anxious people to escape will be able to alleviate the formation of the arching queue and then clear away the stranding at the bottleneck.

\section{Surveillance for the Crowd Density}

In any event gathering phenomena appear because of the large number of participants. The effective monitoring is regarded as a significant premise of security guarantee. It is vital to get the information about the characteristics of participants, activity facilities status, the security arrangement and the surrounding environment, especially the data from important areas such as stairways, exits, entrances, narrow passages, or the convergence of two opposite crowd flows etc., where trickle incidents might trigger terrible accidents. So intensive monitoring needs to be conducted to provide real-time parameters (the crowd density and speed in monitoring areas) by tracking and analyzing the moving objects' behaviors.

\subsection{Crowd Density Estimation}

Crowd density is usually characterized by the number of persons accommodated in per unit area. The greater crowd density usually means the higher degree of population aggregation and also the larger security risk of the throng. Population density mainly relies on intelligent video surveillance system for real-time monitoring of groups, shown as Figure 1.

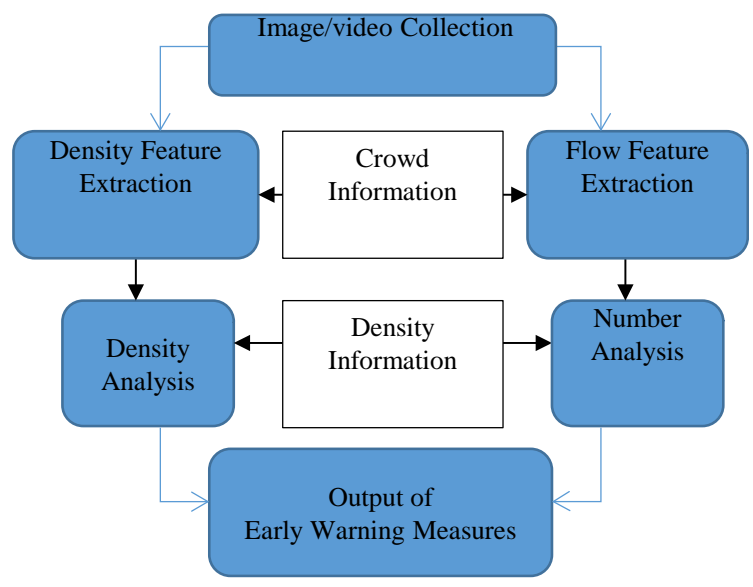

Figure 1. Early warning output depending on the density information. 


\section{1) Statistical Characteristics of the Pixel.}

Statistical modeling of the pixels, an intuitive and effective method, is the first to be adopted to identify population density. The basic idea of the pixel statistical characteristics is that the greater crowd density indicates the denser foreground pixels and edge ones extracted from the pictures, and the larger occupation proportion in the images. Statistical pixel modeling consists of three parts: video collection, feature analysis, and density estimation. Video data is segmented into foreground and background portions by statistical modeling of the pixels after the foreground images about the crowd area are obtained. A statistical model of the background is built for each pixel, and each pixel in an incoming video frame is compared with the background statistical model for that pixel. Pixels are determined to be foreground or background based on the comparisons. Three kinds of characteristic information can be extracted from the foreground image: background feature, edge feature and foreground feature. The background feature is the proportion of the background pixels, the edge feature is the edge length of the crowd area, and the foreground feature is the proportion of the whole image. The extracted background features, edge features and foreground features are placed into a neural network trained in advance to conduct crowd density estimation and analysis.

Statistical modeling of the pixels is characterized by low complexity, wide application range, and accuracy rate of more than 90\% under fixed scenarios. However, the error rate is relatively higher in the scenes of high crowd density, so parameters need to be adjusted to adapt different scenarios while monitoring large samples in a small scope.

\section{2) Texture Analysis.}

The individual quality problem in high-density crowd can be solved by texture analysis which is characterized by high accuracy. In texture analysis method, videotapes are classified and compared for feature extraction. But it cannot fit the low and high population density estimate at the same time, and the huge amount of calculation is not conducive to a real-time video surveillance model.

Feature extraction based on texture information and pixel statistics each has its own applications. Equipments and technologies are influential to reduce the adverse influence of hardware and software when using these conventional methods. More effective crowd feature extraction methods, accuracy rate and system complexity under the consideration of different scenarios are being further developed for implementing an intelligent video surveillance system.

\subsection{Crowd Density Grading}

The different states of the crowds in the context of various congestion densities were described in the previous literatures. Integrated with the relative regulations and practice experience from measuring the movement capability of persons in the area of 1 square meters, crowd density can be graded into 4 classes, as summarized in Table 1. The " $\mathrm{d}$ " in Table 1 represents the crowd density, and correspondingly $d_{i}$ and $d_{o}$ mean the crowd density indoor and outdoor respectively. 
Table 1. State description of different crowd density (Unit: persons $/ \mathrm{m}^{2}$ ).

\begin{tabular}{ccc}
\hline $\begin{array}{c}\text { Crowd Density } \\
\text { Grade }\end{array}$ & $\begin{array}{c}\text { Crowd Density Range } \\
\text { (Indoor; Outdoor) }\end{array}$ & Status Description \\
\hline I & $\mathrm{d}_{\mathrm{i}} \geq 2.8 ; \mathrm{d}_{\mathrm{o}} \geq 3.8$ & Higher Security risk; serious emergency \\
II & $1 \leq \mathrm{d}_{\mathrm{i}}<2.8 ; 1.33 \leq \mathrm{d}_{\mathrm{o}}<3.8$ & Overcrowding; too dangerous \\
III & $0.54 \leq \mathrm{d}_{\mathrm{i}}<1 ; 0.72 \leq \mathrm{d}_{\mathrm{o}}<1.33$ & Interaction; congestion sprouts; controllable \\
IV & $\mathrm{d}_{\mathrm{i}}<0.54 ; \mathrm{d}_{\mathrm{o}}<0.72$ & Crowds move freely, slowly, and smoothly \\
\hline
\end{tabular}

The corresponding measures and risk plans about crowd management are consistent with the crowd density grades.

\section{Strategies for Crowd Management}

The effectiveness of the crowd management depends on good arrangement in multi-aspects such as prejudgment on the participants, supervision of potential threats, the sufficiency of contingency plans, execution capacity and strength of the organizers, the ability of public communication and information communication, reasonable division and arrangement for field staff, the reasonable sign design, and services for special groups including the old, children, illness or disabled people and pregnant women, etc.

\subsection{Understanding the Participants in an Event}

1) Predicting the Capacity of Each Site to Accommodate People.

Any site has its limited capacity to accommodate people. Disasters may happen when the capability is exceeded. The capacity that a venue can bear refers not only to the total capacity of certain particular sites, some bottleneck areas where accidents usually happen, or some specific fixed or temporary equipments, but also the endurance management and command ability of relative security personnel. The video surveillance and sensor system can be utilized to monitor the inflow, outflow and average density and ensure the crowd density is within a permitted range.

\section{2) Considering the Composition of Participants.}

The composition of participants is a significant factor to influence the stability of public activities. Participants are distinct from each other in characteristics, education levels, and personal experiences, and so on; what's more, in various events the composition of them is complex and different. Forecasting composition of participants in advance can reinforce the pertinence of management plans and the effectiveness of contingency plans, and permanently, help deal with contradictions caused by cultural difference between the participants from diverse background. In addition the activity organizers are able to provide better services in such aspects as traffic, food and water, etc., and take more suitable preventive measures if they are informed the features of participants ahead of time. Such approaches as building up audience management system according to the data from previous years, conducting surveys online or offline, implementing register system in the process of ticket purchase are beneficial to obtain the 
information of possible participants.

\section{3) Forecasting the Distribution of Participants.}

The time and space distribution of participants in an event can be estimated by historical statistics and mathematical calculation, include the regular of crowd change and the distribution of people. Such factors as seasonal change, the features of activities and the habitants' culture may have a great impact on the time distribution of participants while the arrangement of activities influences their space distribution. People's possible gathering places, coverage area and the duration of their gathering can be predicted in advance to avoid some peak time, focus on the bottleneck places and arrange security measures better.

\subsection{Early Warning Mechanism in Crowd Security Management}

Early warning system, an intelligent system runs automatically and notifies management personnel of site conditions according to the monitoring data, is an anti-risk program to create an alert for corresponding measures taken in advance. Early warning information is issued by different levels of risk, and then to develop appropriate control plans. According to the state of different risk levels calculated by crowd density in Table 1, early warning levels and the corresponding suggestions and measures can be summarized as Table 2 .

\section{1) Plan for Risk Level IV.}

For risk level 4, crowds move smoothly and security personnel are not needed to take strict control measures, but only a small number of them are needed to patrol the scene, arrange and guide posts reasonably, and pay attention to changes of the crowd.

\section{2) Plan for Risk Level III.}

As for risk level 3, more people throng into the notable areas, congestion appears at times, and certain kinds of danger sprout. However, the situation is still controllable, and the risk is barely acceptable. In this situation, evacuation is advised, and police and security personnel are setting out to deal with the congestion in the crowd areas.

\section{3) Plan for Level Risk II.}

At the risk level 2 the avenue is overcrowding. People are moving by the involuntary push. The pressure from the interaction with others can be experienced. Situation is dangerous and communication with the crowd becomes crucial to avoid any incidents. All the stakeholders integrate to take part in evacuation guidance.

Table 2. Plan for different risk levels.

\begin{tabular}{ccr}
\hline Risk levels & Warning signs & Plan for different risk levels \\
\hline I & Red & $\begin{array}{r}\text { Additional and sufficient emergency vehicles and manpower are } \\
\text { ensured to come to the rescue. }\end{array}$ \\
II & Orange & $\begin{array}{r}\text { Communication becomes crucial; all stakeholders take part in } \\
\text { evacuation guidance. }\end{array}$ \\
III & Yellow & Evacuation is advised. The congestion is being disposed. \\
IV & Green & Security personnel are needed to patrol the scene. \\
\hline
\end{tabular}




\section{4) Plan for Level Risk I.}

The possibility of security risk regarded as level 1 is much higher. All the people in the site should be evacuated safely and orderly in a very limited time. Mandatory evacuation is carrying out. Calm down the crowd and guide it. Continue to evacuate people. Additional and sufficient emergency vehicles and manpower are ensured to come to the rescue.

\subsection{Crowd Diversion Measures}

Depending on the crowd density surveillance, early warning mechanism, prediction procedures and the data about the crowds are collected and need to be informed the staff involved in the event.

\section{1) Choosing reasonable routes.}

When choosing routes to separate the flow, the stationary flow and the capacity of facilities to contain people, as well as some specific condition (weather, types of emergencies, and road conditions) should be considered. Remove the unreasonable section to avoid the obstacles that may affect evacuation performance. Weak infrastructure is one of the reasons that reduce the crowd capacity and slow the speed. Both disorderly infrastructure and unreasonable distribution increase the risk in an event. They also hinder the evacuation and rescue process when an incident occurs. Neat streets and convenient traffic are the secondary condition to keep participants safe. Smooth traffic can be guaranteed by adding striking road maps, planning business streets and stalls, controlling peddlers and setting up service areas. Medical facilities and professional staffs should be provided as a routine work.

\section{2) Releasing information timely.}

Informing participants of the evacuation guidance at all times and releasing information about the site conditions in time has been always crucial in any event. Not a few disasters illustrated that people at the back of the queue couldn't get any idea of what happened at the front and take unsuitable actions such as push and shove, precipitate them into tension, etc., which finally led into grave consequence. Also, as mentioned above, most people tended to sink into the panic and be at a loss in emergency, and just follow others without response to all the information in the site. Therefore, notifying people of routes, cues and signs at exits and entrances and other information can evade their misunderstanding about the situation, provide them useful message and prompt them to make correct decisions. Moreover, depending on the forecast data, organizers can give participants some advice to influence their choice including the time and route to go out, the members, routes and items to visit, etc., so that the gathering pressures at the same places and at the same time can be relieved.

\section{An Example}

Take a metro station of China as an example. With the improvement of urban traffic, subway has become a competitive choice for people to travel owing to its advantage of convenience and efficiency. Some subway stations maintain high- 
traffic throughout the year and the passenger flow significantly increases particularly in the holidays, which leads to the crowd aggregation in a high degree and stampede accidents or other disasters prone to happen.

Effective risk assessment and early warning mechanisms are necessary to pre-control the possible disaster triggers in busy subway stations. First, it is essential to estimate and control the maximum crowd flow limit of evacuation passages. The capacity of each site should be predicted to estimate its limitation. The bottleneck areas also need to be considered because a jam arises from competitive behaviors. Meanwhile, whether the exit has doors or not also has influence on the stock and flow of crowds. The structure of the spot can be changed to fit the participants to move freely. Second, it is deemed that crowd density, the speed of movement and people's psychology all have critical impacts on evacuation efficiency. Crowd in an area where each person cover less than 0.26 $\mathrm{m}^{2}\left(\mathrm{~d}_{\mathrm{o}} \geq 3.8\right.$ persons $\left./ \mathrm{m}^{2}\right)$ may be dangerous to lead to squeeze, trip and casualties. So crowd density is supervised in real time and reliable information is released to crowds in time to avoid the loss of rational behaviors. Third, it's important to calculate the composition of participants according to the entrance ticket information system. Environment factors such as temperature, sound and light are created to comfort people's psychological reaction. Last but not least, organizers who know the spot structures clearly should be effective, and suitable and experienced guards, safety equipments and evacuation passages be ensured to evacuate the participants.

According to the state description of different density levels in Table 1, early warning information about the metro stations is issued and appropriate control plans are developed by different risk levels. For Risk Level IV under which queuing phenomena with no stranding exist but do not affect the order, the security staff need stick to their positions in the station hall, platforms, passages or other sites, observe and pay attention to the changes of the passenger flow and strengthen the guidance when necessary. If a little stagnancy emerges but without congestion in the station, the situation of which can be regarded as Risk Level III, then passenger traffic conditions at platforms, gates and stagnant spots need to be paid close attention to. The station personnel are mobilized and arranged to support the "dredging". Close the entrance and prevent the continuous influx of passengers when passengers' stranding becomes serious. With more congestion, the spot becomes overcrowded and stagnancy phenomena arise among passengers. This situation at Risk Level II is "unacceptable", under which the security management should be centered on "flow restriction and crowd guidance". Emergency responding teams are set up to carry out security work; metro security staff and subway duty personnel joint to explain to and guide passengers; and additional spare trains are arranged to transport more passengers out of the station sites. In the case of serious congestion at Risk Level I, people must be evacuated out of the spot safely within a very limited time. Professional security staffs make up emergency support teams to deal with on-site guidance, broadcasting at the station and on the train inform the pas- 
sengers, and evacuation is carried out in time.

To match up the early warning mechanism, the following measures may be developed as the routine security work in order to be able to evacuate passengers as soon as possible in emergencies.

1) Set the ground ticket office, use the ancillary metal detectors in the security system to speed up the screening rate, open the spare trains during peak hours and reduce the interval between vehicles in order to transport passengers out of the stations quickly.

2) Restrict the passenger flow when necessary. Set the spiral-shaped diversion railings to control the access speed and ease passengers' irritable mood. And even adjust some exits only for outbound.

3) Signs and broadcasting are set and on-site staff are arranged to guide passengers to enter and leave the station hall quickly. Volunteers are welcomed to help evacuate passengers.

4) Security staff are trained to enhance risk awareness and improve service capability, public are educated to avoid the uncivilized behaviors by posting posters or distributing leaflets. Develop regulations to prohibit the marketing activities near some busy subway stations on holidays.

5) Prepare risk plans for large passenger flow.

\section{Conclusion}

In any event, all stakeholders are amenable to form risk consciousness and grasp the basic knowledge and skills to avoid risk, protect themselves and evacuate properly and promptly in an event. The elaborated risk plan should be studied out in advance to ensure the information about the crowds, ways and procedures in different situations notified to everyone involved in the event. It is extremely indispensable to remove all hidden threats when preparing for the event, and communicate with then control the crowd efficaciously during the process of the event. When confronted with emergencies, enforcers need to learn to keep calm and implement evacuation plans professionally and scientifically to ensure the crowd to choose suitable routes or safe destinations and carry out suitable behaviors consistent with the risk plans.

\section{Acknowledgements}

This work is supported by the Major Program of the National Social Science Foundation of China (Grant No. 15AGL021) and the Fundamental Research Funds for the Central Universities (Grant No. CSY16004). The author also gratefully acknowledges the helpful comments and suggestions of the reviewers and the editors.

\section{References}

[1] Bjelland, H., Njå, O., Heskestad, A.W., et al. (2014) The Concepts of Safety Level and Safety Margin. Fire Technology, 51, 409-441.

https://doi.org/10.1007/s10694-014-0400-y 
[2] Helbing, D. and Mukerji, P. (2012) Crowd Disasters as Systemic Failures: Analysis of the Love Parade Disaster. EPJ Data Science, 1, 1-40. https://doi.org/10.1140/epjds7

[3] Meacham, B.J., Dembsey, N.A., Schebel, K., et al. (2012) Use of Small-Scale Test Data to Enhance Fire-Related Threat, Vulnerability, Consequence and Risk Assessment for Passenger Rail Vehicles. Journal of Homeland Security \& Emergency Management, 9, 1-16. https://doi.org/10.1515/1547-7355.1850

[4] Challenger, R., Clegg, C. and Robinson, M. (2009) Understanding Crowd Behaviours. UK Cabinet Office, York.

[5] Wijermans, N., Conrado, C. and Steen, M.V. (2016) A Landscape of Crowd-Management Support: An Integrative Approach. Safety Science, 86, 142-164. https://doi.org/10.1016/j.ssci.2016.02.027

[6] Zhou, B., Tang, X. and Wang, X. (2015) Learning Collective Crowd Behaviors with Dynamic Pedestrian-Agents. International Journal of Computer Vision, 111, 50-68. https://doi.org/10.1007/s11263-014-0735-3

[7] Omodei, E., Bazzani, A., Rambaldi, S., et al. (2014) The Physics of the City: Pedestrians Dynamics and Crowding Panic Equation in Venezia. Quality and Quantity, 48, 347-373. https://doi.org/10.1007/s11135-012-9773-5

[8] Chu, M.L., Parigi, P., Latombe, J.C., et al. (2015) Simulating Effects of Signage, Groups, and Crowds on Emergent Evacuation Patterns. Ai \& Society, 30, 493-507. https://doi.org/10.1007/s00146-014-0557-4

[9] Castro, F.E.V.G. and Pabico, J.P. (2015) Microsimulations of Arching, Clogging, and Bursty Exit Phenomena in Crowd Dynamics. Proceedings of the 10 th National Conference on Information Technology Education (NCITE 2012), Laoag, 18-20 October 2012. http://de.arxiv.org/pdf/1506.07781

\section{Submit or recommend next manuscript to SCIRP and we will provide best} service for you:

Accepting pre-submission inquiries through Email, Facebook, LinkedIn, Twitter, etc. A wide selection of journals (inclusive of 9 subjects, more than 200 journals)

Providing 24-hour high-quality service

User-friendly online submission system

Fair and swift peer-review system

Efficient typesetting and proofreading procedure

Display of the result of downloads and visits, as well as the number of cited articles

Maximum dissemination of your research work

Submit your manuscript at: http://papersubmission.scirp.org/

Or contact eng@scirp.org 